\title{
Bright octave-span mid-IR supercontinuum generation in silicon germanium waveguide
}

\begin{abstract}
Alberto Della Torre, Milan Sinobad, Barry Luther-Davies, Pan Ma, Stephen Madden, et al.
\end{abstract}

Alberto Della Torre, Milan Sinobad, Barry Luther-Davies, Pan Ma, Stephen Madden, Sukanta Debbarma, Khu Vu, David J. Moss, Arnan Mitchell, JeanMichel Hartmann, Jean-Marc Fedeli, Christelle Monat, Christian Grillet, "Bright octave-span mid-IR supercontinuum generation in silicon germanium waveguide," Proc. SPIE 10921, Integrated Optics: Devices, Materials, and Technologies XXIII, 109210A (4 March 2019); doi: 10.1117/12.2517866 


\title{
Bright octave-span mid-IR supercontinuum generation in silicon germanium waveguide
}

\author{
Alberto Della Torre* ${ }^{* a}$, Milan Sinobad ${ }^{\mathrm{a}, \mathrm{b}}$, Barry Luther-Davies ${ }^{\mathrm{c}}$, Pan Ma ${ }^{\mathrm{c}}$, Stephen Madden ${ }^{\mathrm{c}}$, Sukanta \\ Debbarma $^{\mathrm{c}}$, Khu Vu ${ }^{\mathrm{c}}$, David J. Moss ${ }^{\mathrm{d}}$, Arnan Mitchell ${ }^{\mathrm{b}}$, Jean-Michel Hartmann ${ }^{\mathrm{e}}$, Jean-Marc Fedeli ${ }^{\mathrm{e}}$, \\ Christelle Monat ${ }^{\mathrm{a}}$, Christian Grillet ${ }^{\mathrm{a}}$ \\ ${ }^{a}$ Université de Lyon, Institut des Nanotechnologies de Lyon (INL), 69131 Ecully, France ; ${ }^{\text {bSchool }}$ \\ of Engineering, RMIT University, Melbourne, VIC 3001, Australia; ' Laser Physics Center, \\ Australian National University, Canberra, ACT 0100, Australia; ${ }^{\mathrm{d} C e n t r e}$ for Microphotonics, \\ Swinburne University of Technology, Hawthorn, VIC 3122, Australia; ${ }^{e}$ Université Grenoble \\ Alpes,CEA-Leti, 38054 Grenoble Cedex 9, France; *e-mail : alberto.della-torre@ec-lyon.fr
}

\begin{abstract}
We present silicon-germanium on silicon waveguides as a suitable platform for on-chip supercontinuum generation in the mid-infrared. We report low propagation loss $(<0.4 \mathrm{~dB} / \mathrm{cm})$ in the $3.5-5 \mu \mathrm{m}$ range, leading to an octave spanning supercontinuum extending up to $8.5 \mu \mathrm{m}$ with a high average power of more than $10 \mathrm{~mW}$ on-chip. Furthermore, we present the addition of a chalcogenide cladding layer as a simple post-processing technique to fine tune the waveguide dispersion which, in turn, governs the properties of the generated supercontinuum.
\end{abstract}

Keywords: Supercontinuum generation; nonlinear optics; integrated optics; mid-infrared; dispersion trimming

\section{INTRODUCTION}

Supercontinuum (SC) sources are of great interest in a wide variety of fields due to their high power spectral density over a broad bandwidth. In particular, on-chip SC generation in the mid-infrared (mid-IR), due to the strong absorption lines of many chemical compounds in this spectral region, is a key element for applications in biomedical imaging, medical and environmental sensors, food quality analysis and security [1,2]. Wide mid-IR bandwidth SC were first demonstrated in fibers [3-7], in a chalcogenide based platform [8] and recently in CMOS compatible integrated platforms [9-12]. Among the different CMOS compatible platforms that have been proposed for mid-IR SC generation, silicon-germanium on silicon waveguides are very promising thanks to their wide transparency window [1], low loss [13-16] and good nonlinear properties [17]. Here we present our recent progress on SC generation in step index $\mathrm{Si}_{0.6} \mathrm{Ge}_{0.4} \mathrm{Si}$ waveguides. We report octave spanning SC generation up to $8.5 \mu \mathrm{m}$, the longest wavelength so far achieved in any integrated platform. Moreover, we demonstrate anomalous-to-normal dispersion shift by adding a chalcogenide cladding layer, introducing a simple technique for post-processing dispersion trimming.

\section{SUPERCONTINUUM GENERATION}

\subsection{Waveguides design}

In order to achieve SC generation through a soliton fission process, we designed our waveguide to operate in a low and relatively flat anomalous dispersion regime. We investigated a $7 \mathrm{~cm}$ long $\mathrm{Si}_{0.6} \mathrm{Ge}_{0.4} \mathrm{Si}$ waveguide with a top air-cladding (see fig. 1 inset) in order to achieve low anomalous dispersion despite the low core-cladding index contrast ( $\sim 0.16)$ [18]. The key parameters of the waveguide were calculated by a finite difference mode solver. Fig. 1 shows the group velocity dispersion (GVD) profile and the mode confinement of the fundamental transverse-magnetic (TM) mode. A large cross section of $6 \mu \mathrm{m} \times 4.2 \mu \mathrm{m}$ was chosen in order to extend the cutoff wavelength up to $9.3 \mu \mathrm{m}$, enabling us to exploit, in principle, the full transparency window of the SiGe/Si platform. The cutoff shift was obtained at the cost of a large crosssectional area and multimode operation at the pump wavelength $(4.15 \mu \mathrm{m})$. The large cross-sectional area allowed to achieve $98 \%$ of light confinement in the waveguide core at the pump wavelength. Most importantly, the dispersion of the

Integrated Optics: Devices, Materials, and Technologies XXIII, edited by Sonia M. García-Blanco, Pavel Cheben, Proc. of SPIE Vol. 10921, 109210A - @ 2019 SPIE · CCC code: 0277-786X/19/\$18 - doi: 10.1117/12.2517866 
TM fundamental mode was low and anomalous across a wide spectral range, with two zero dispersion wavelengths at 4.5 $\mu \mathrm{m}$ and $7.7 \mu \mathrm{m}$, which helped to maximize the bandwidth of the SC.

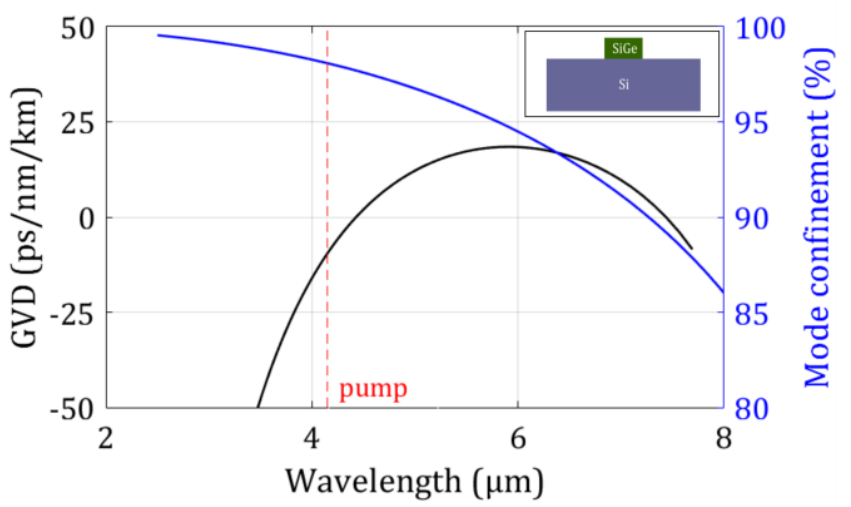

Figure 1. Calculated group velocity dispersion (GVD) (black curve) and mode confinement (blue curve) of the fundamental TM mode of the $\mathrm{Si}_{0.6} \mathrm{Ge}_{0.4} \mathrm{Si}$ with $6 \mu \mathrm{m}$ x $4.2 \mu \mathrm{m}$ cross-section. The red dashed line indicates the pump wavelength (4.15 $\mu \mathrm{m})$. The top-right inset shows a schematic of the waveguide.

\subsection{Propagation loss}

We measured the propagation losses using the cutback method by probing waveguides with three different lengths each (from 2 to $7 \mathrm{~cm}$ ) under relatively low average powers $(<1 \mathrm{~mW})$. Measurements were performed using a tunable OPA delivering $7.5 \mathrm{ps}$ long pulses at a $1.5 \mathrm{MHz}$ repetition rate across a tunable wavelength range between approximately 3-5 $\mu \mathrm{m}$. The measured propagation loss of the fundamental TM mode (fig. 2) was relatively flat versus wavelength, and reached a value as low as $0.23 \mathrm{~dB} / \mathrm{cm}$ around $4.75 \mu \mathrm{m}$. By paying the price of a higher modal effective area, the large waveguide cross-section allowed to reach record-low loss for mid-IR integrated platforms.

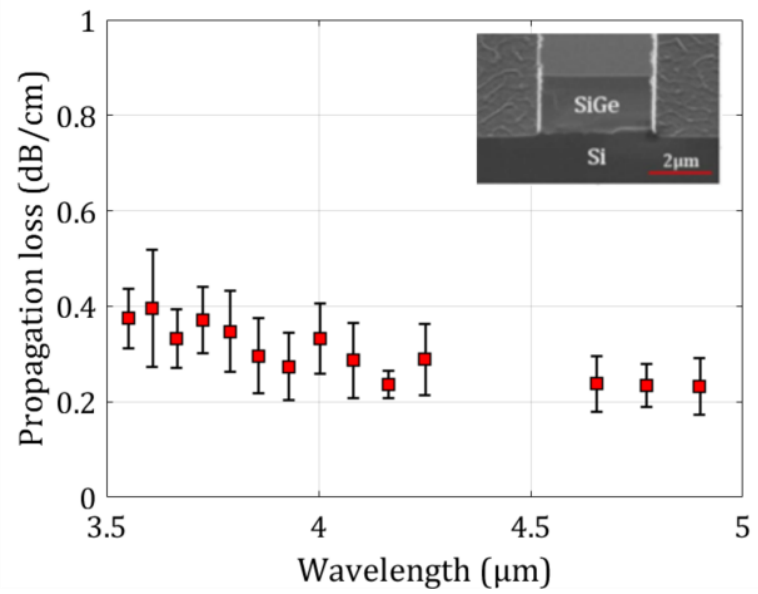

Figure 2. Measured propagation loss for the fundamental TM mode. Inset: 3D scanning electron microscopy image of a cleaved $\mathrm{SiGe} 40 \% / \mathrm{Si}$ waveguide (adapted from [18]).

\subsection{Supercontinuum generation}

The waveguide was pumped in the TE polarization by a $200 \mathrm{~mW}$ tunable OPA laser (MIROPA-fs, Hotlight Systems) delivering $\approx 200 \mathrm{fs}$ pulses centered at $4.15 \mu \mathrm{m}$, with a repetition rate of $63 \mathrm{MHz}$. The pump wavelength was selected to operate as close as possible to the zero- dispersion wavelength (ZDW), while avoiding the 4.15-4.30 $\mu \mathrm{m} \mathrm{CO}_{2}$ absorption wavelength band. At a coupled peak power of $3.54 \mathrm{~kW}(25 \mathrm{~mW}$ coupled average power) the generated 1.4 octave-wide 
SC (see fig. 3) extended from 3 up to $8.3 \mu \mathrm{m}$, thus covering great part of the molecular fingerprint band. The extension of the SC towards longer wavelengths was limited by the silicon absorption in the substrate. The generated spectrum was both spectrally flat, as evidenced by the large $-10 \mathrm{~dB}$ bandwidth of $4.9 \mu \mathrm{m}$ (from 3.1 to $8 \mu \mathrm{m}$ ), which is comparable with the $-30 \mathrm{~dB}$ bandwidth of $5.3 \mu \mathrm{m}$, and spectrally bright, with a $12.5 \mathrm{~mW}$ on-chip power (taking into account the $\sim-5 \mathrm{~dB}$ coupling loss at the input/output facet).

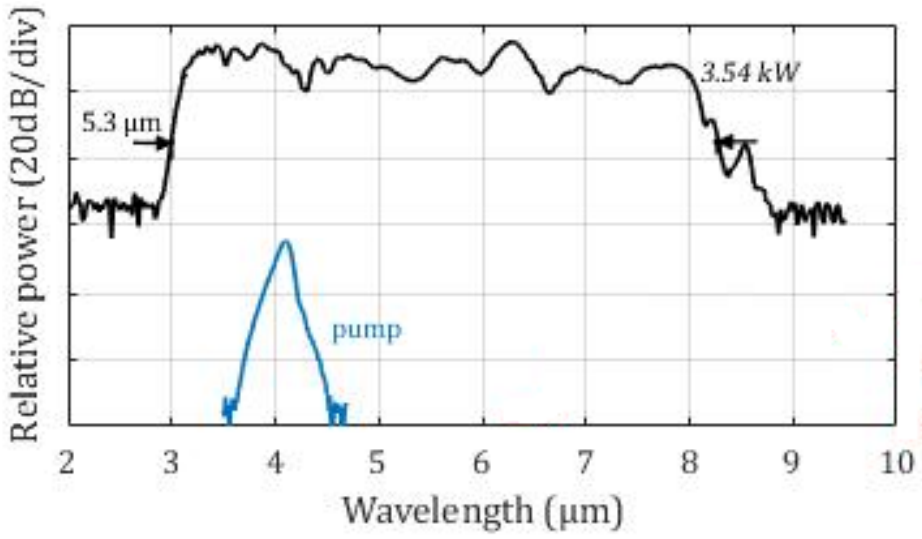

Figure 3. Spectra measured out of the $7 \mathrm{~cm}$ long waveguide pumped in TM polarization at $4.15 \mu \mathrm{m}$ and $3.54 \mathrm{~kW}$ of coupled peak power. The black arrows highlight the $-30 \mathrm{~dB}$ bandwidth of $5.3 \mu \mathrm{m}$ (adapted from [18]).

\section{DISPERSION TRIMMING IN A HYBRID CHALCOGENIDE/SILICON-GERMANIUM WAVEGUIDE}

We have demonstrated SC generation in a SiGe/Si waveguide covering the molecular fingerprint band. This result was achieved by mean of a careful design of the waveguide and of its group velocity dispersion. However, the dispersive properties are set at the design stage and cannot be adjusted once the device has been fabricated. Hence, they are subject to fabrication inaccuracies, surface contamination and presence of defects. Post-process tuning mechanisms are therefore of great interest to adjust or correct a-posteriori the waveguide dispersion to match the target value. Several approaches have been proposed for post-process tuning of optical properties in optical fibers [19,20] and photonic crystals [21-24]. However, the restricted number of design parameters makes post-process dispersion control of waveguides trickier. In this context, we reported a proof of concept demonstration in which the deposition of a chalcogenide top-cladding on nonlinear $\mathrm{SiGe} / \mathrm{Si}$ waveguides enabled us to tune the group velocity dispersion of the fundamental mode [25]. This demonstrates that the heterogeneous integration of materials can be used as a post-processing technique to tune the waveguide dispersive properties and therefore control SC generation.

\subsection{Chalcogenide clad waveguide}

A second waveguide, with a $3.75 \mu \mathrm{m} \times 2.70 \mu \mathrm{m}$ cross-section and operating in the low anomalous dispersion regime, was studied. This design was chosen in order to have transverse-electric (TE) single-mode operation. The drawback of this design is the relatively short $(\sim 6 \mu \mathrm{m})$ cutoff wavelength that intrinsically limits the SC spectrum at long wavelengths.

After the supercontinuum generation in the air clad case, the waveguide was coated with a $1.26 \mu \mathrm{m}$ thick layer of a chalcogenide $\mathrm{Ge}_{11.5} \mathrm{As}_{24} \mathrm{Se}_{64.5}$, deposited by thermal evaporation [26]. The chalcogenide refractive index was considered constant and equal to 2.6 [27]. Fig. 4(a) shows a schematic of the waveguide before and after the deposition of the chalcogenide, while fig. 4(b) and 4(c) show respectively a scanning electron microscope (SEM) and atomic force microscope (AFM) image of the waveguide surrounded by the chalcogenide layer. Even though the influence of the cladding on the linear optical properties of the waveguide was not experimentally investigated, numerical simulations, taking into account the roughness measured by AFM $(\mathrm{rms} \approx 15 \mathrm{~nm})$ at both the core/chalcogenide cladding and top chalcogenide interfaces, showed that the impact of scattering is negligible $(\sim 0.2 \mathrm{~dB} / \mathrm{cm}$ of extra loss $)$. 


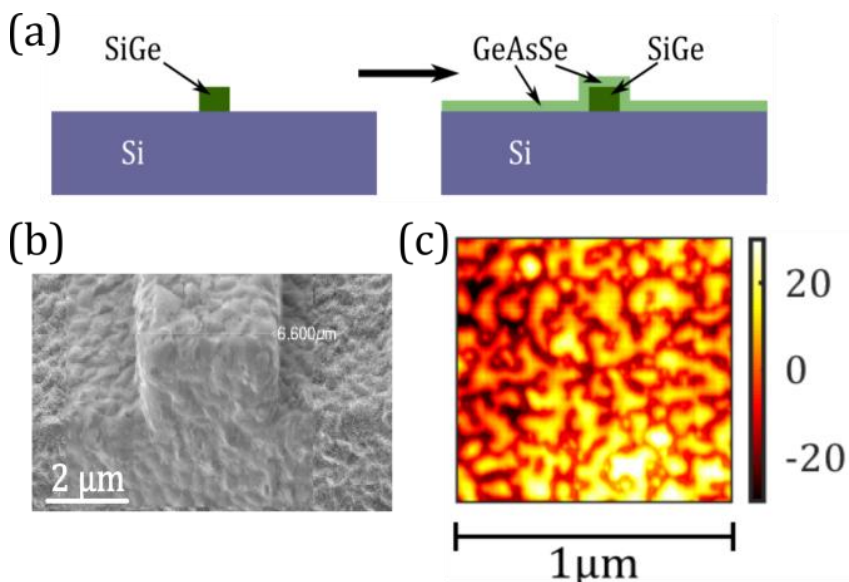

Figure 4. (a) Schematic of the waveguide before and after the deposition of the chalcogenide clad. SEM (b) and AFM (c) images of the waveguide after the deposition of chalcogenide. (adapted from [25]).

Fig. 5 shows the group velocity dispersion profile and the spectral dependence of the effective area with and without the chalcogenide layer. At the pump wavelength $(4.15 \mu \mathrm{m})$ the dispersion shifted from anomalous to normal when the chalcogenide cladding was added. Furthermore, due to the low refractive index difference between the core and top cladding, the effective area of the mode slightly increased by $\sim 10 \%$ when the chalcogenide cladding was added; however, the mode confinement in the core of the waveguide remained unaffected and high $(\sim 94 \%)$.

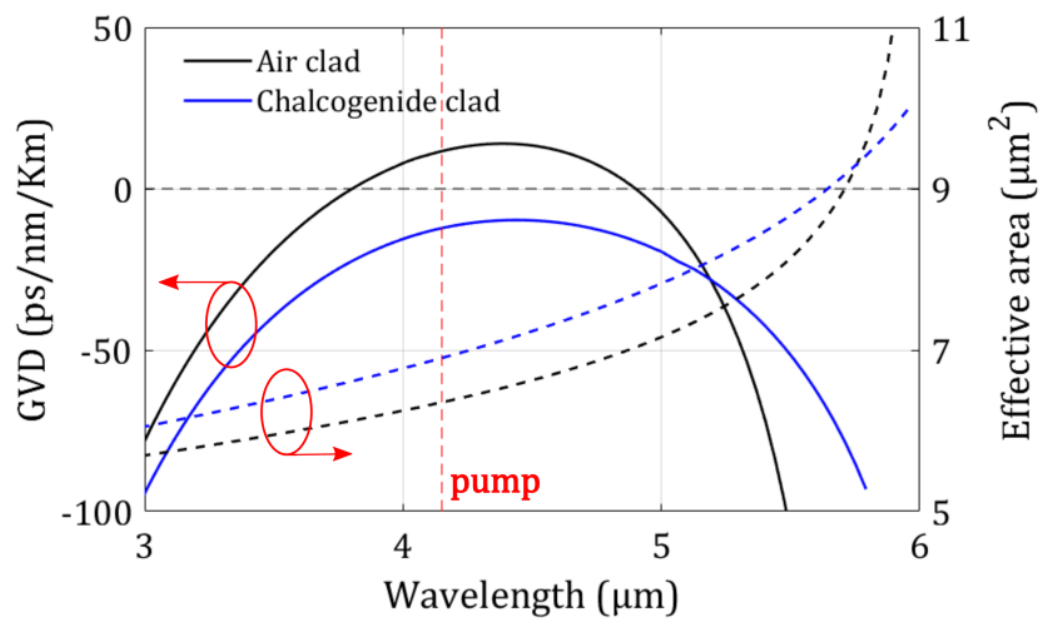

Figure 5. (a) Calculated GVD (continuous curves) and effective area (dashed curves) for air clad (black) and chalcogenide clad (blue) waveguide. The red dashed line indicates the pump wavelength $(4.15 \mu \mathrm{m})$.

\subsection{Supercontinuum generation}

Fig. 6 shows the experimental (blue) and simulated (red) output spectra at $2.35 \mathrm{~kW}$ coupled peak power (corresponding to up to $50 \mathrm{~mW}$ of laser average power) obtained by pumping the waveguide before (left) and after (right) the deposition of the chalcogenide cladding layer. Some differences can be observed, which illustrate the changes to the dispersion caused by the addition of the chalcogenide top cladding. In the former case, the uneven amplitude across the spectrum (with a $-30 \mathrm{~dB}$ bandwidth of $3.55 \mu \mathrm{m}$ ) is typical of SC generation in anomalous dispersion regime (driven by soliton fission). In the latter case, the narrower and smoother spectrum (with a $-30 \mathrm{~dB}$ bandwidth extending from 3.1 to $5.5 \mu \mathrm{m}$ ) is typical of SC generation in normal dispersion regime (driven by self-phase modulation and optical wave breaking). 

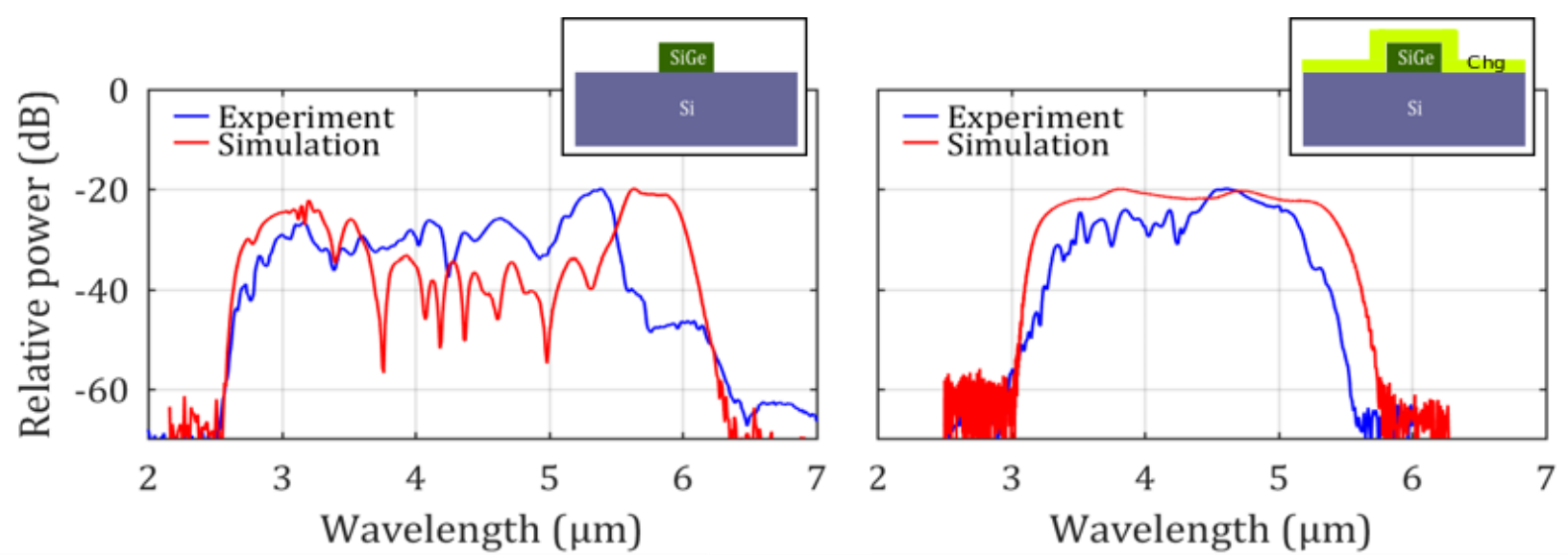

Figure 6. Calculated (red) and measured (blue) spectra out of the air cladded (left) and chalcogenide cladded (right) SiGe/Si waveguide with the same $3.75 \times 2.7 \mu \mathrm{m}^{2} \mathrm{SiGe}$ core cross-section. The insets show a schematic of the waveguide's cross section in the two cases.

\subsection{Dispersion tailoring}

We have experimentally demonstrated that the addition of a chalcogenide layer on top of a $\mathrm{SiGe} / \mathrm{Si}$ waveguide leads to a change in the dispersion, shifting, in our particular example, from an anomalous to normal dispersion. In order to clarify the impact of the chalcogenide thickness on the dispersion GVD profile, we have numerically calculated the dispersion curve for four different thicknesses, from 0.25 to $1.26 \mu \mathrm{m}$ (see Fig. 7). As the chalcogenide thickness increases, the overall dispersion gradually decreases, eventually reaching normal values for thicknesses higher than $500 \mathrm{~nm}$. Moreover, the dispersion profiles converge as the thickness approaches $1 \mu \mathrm{m}$, in agreement with the confinement of the mode in the waveguide core. Along with the dispersion shift, a variation of the zero dispersion wavelengths and a flattening of the profile take place. As a flat profile of the dispersion is generally targeted for SC generation, both in the anomalous and in the normal dispersion regime, the possibility of controlling the dispersion profile and the position of the ZDWs by simply changing the thickness of the chalcogenide layer (with a reasonable resolution of around $100 \mathrm{~nm}$ ) is a convenient post-process dispersion engineering tool.

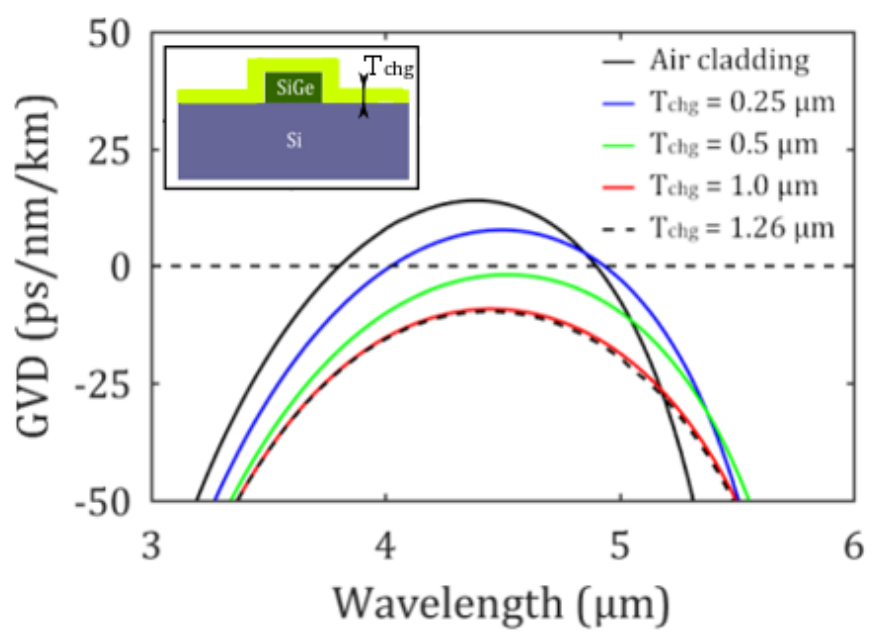

Figure 7. Calculated group velocity dispersion (GVD) for different thicknesses of the chalcogenide layer. The inset shows a schematic of the waveguide (adapted from [25]).

\section{CONCLUSION}

In conclusion, we have summarized our recent work on SC generation in silicon germanium waveguides. A careful waveguide design allowed us to generate an octave spanning SC up to $8.5 \mu \mathrm{m}$. We have demonstrated that it is possible 
to fine tune the waveguide dispersion by adding a chalcogenide cladding, introducing a simple tool for post-processing dispersion control. These results harness silicon-germanium on silicon platforms as a suitable platform for on-chip SC generation covering the molecular fingerprint region, with potential applications in biomedical imaging, medical and environmental sensors, food quality analysis and security.

\section{REFERENCES}

[1] Soref, R., "Mid-infrared photonics in silicon and germanium," Nat. Photonics 4, 495-497 (2010).

[2] Zhang, L., Agarwal, A. M., Kimerling, L. C. and Michel, J., 3Nonlinear Group IV photonics based on silicon and germanium: From near-infrared to mid-infrared," Nanophotonics 3, 247-268 (2014).

[3] Petersen, C. R., Møller, U., Kubat, I., Zhou, B., Dupont, S., Ramsay, J., Benson T., Sujecki, S., Abdel-Moneim, N., Tang, Z., Furniss, D., Seddon, A. and Bang, O., "Mid-infrared supercontinuum covering the 1.4-13.3 $\mu \mathrm{m}$ molecular fingerprint region using ultra-high NA chalcogenide step-index fibre," Nat. Photonics 8, 830-834 (2014).

[4] Yu, Y., Zhan, B., Gai, X., Zhai, C., Qi, S., Guo, W., Yang, Z.,Wang, R., Choi, D. Y., Madden, S. and LutherDavies, B., "1.8-10 $\mu \mathrm{m}$ Mid-Infrared Supercontinuum Generated in a Step-Index Chalcogenide Fiber Using Low Peak Pump Power," Opt. Lett. 40, 1081 (2015).

[5] Cheng, T., Nagasaka, K., Tuan, T. H., Xue, X., Matsumoto, M., Tezuka, H., Suzuki, T., and Ohishi, Y., "Midinfrared supercontinuum generation spanning 2.0 to $15.1 \mu \mathrm{m}$ in a chalcogenide step-index fiber," Opt. Lett. 41, 2117 (2016).

[6] Petersen, C. R., Engelsholm, R. D., Markos, C., Brilland, L., Caillaud, C., Trolès, J. and Bang, O., "Increased midinfrared supercontinuum bandwidth and average power by tapering large-mode-area chalcogenide photonic crystal fibers," Opt. Express 25, 15336 (2017).

[7] Hudson, D. D., Antipov, S., Li, L., Alamgir, I., Hu, T., El Amraoui, M., Messaddeq, Y., Rochette, M., Jackson, S. D. and Fuerback, A., "Toward all-fiber supercontinuum spanning the mid-infrared," Optica 4, 1163 (2017).

[8] Yu, Y., Gai, X., Ma, P., Vu, K., Yang, Z.,Wang, R., Choi, D. Y., Madden, S. and Luther-Davies, B., "Experimental demonstration of linearly polarized $2-10 \mu \mathrm{m}$ supercontinuum generation in a chalcogenide rib waveguide," Opt. Lett. 41, 958 (2016).

[9] Lau, R. K. W., Lamont, M. R. E., Griffith, A. G., Okawachi, Y., Lipson, M. and Gaeta, A., L., "Octave-spanning mid-infrared supercontinuum generation in silicon nanowaveguides," Opt. Lett. 39, 4518-21 (2014).

[10] Kou, R., Hatekeyama, T., Horng, J., Kang, J. H., Wang, Y., Zhang, X. and Wang, F., "Mid-IR broadband supercontinuum generation from a suspended silicon waveguide," Opt. Lett. 43, 1387-1390 (2018).

[11] Singh, N., Hudson, D. D., Yu, Y., Grillet, C., Jackson, S. D., Casas-Bedoya, A., Read, A., Atanackovic, P., Duvall, S., G., Palomba, S., Luther-Davies, B., Madden, S., Moss, D. J. and Eggleton, B., J., "Midinfrared supercontinuum generation from 2 to $6 \mu \mathrm{m}$ in a silicon nanowire," Optica 2, 797 (2015).

[12] Nader, N., Maser, D. L., Cruz, F. C., Kowligy, A., Timmers, H., Chiles, J., Fredrick, C., Westly, D. A., Nam, S. W., Mirin, R. P., Shainline, J. M. and Diddams, S., "Versatile silicon-waveguide supercontinuum for coherent midinfrared spectroscopy," APL Photonics 3, 036102 (2018).

[13] Ramirez, J. M., Vakarin, V., Gilles, C., Frigierio, J., Ballabio A., Chaisakul, P., Le Roux, X., Alonso-Ramos, C., Maisons, G., Vivien, L., Carras, M., Isella, G. and Marris-Morini, D., "Low-loss Ge-rich $\mathrm{Si}_{0.2} \mathrm{Ge}_{0.8}$ waveguides for mid-infrared photonics," Opt. Lett. 42, 105 (2017).

[14] Ramirez, J. M., Liu, Q., Vakarin, V., Frigierio, J., Ballabio A., Le Roux, X., Bouville, D., Vivien, L., Carras, M., Isella, G. and Marris-Morini, D.,"Graded SiGe waveguides with broadband low-loss propagation in the mid infrared," Opt. Express 26, 528-531 (2018).

[15] Carletti, L., Ma, P., Yu, Y., Luther-Davies, B., Hudson, D. D., Monat, C., Orobtchouk, R., Madden, S., Moss, D. J., Brun, M., Ortiz, S., Labeye, P., Nicoletti, S. and Grillet, C., "Nonlinear optical response of low loss silicon germanium waveguides in the mid-infrared," Opt. Express 23, 8261 (2015).

[16] Carletti, L., Sinobad, M., Ma, P., Yu, Y., Allioux, D., Orobtchouk, R., Moss, Brun, M., Ortiz, S., Labeye, P., Hartmann, J. M., Nicoletti, Madden, S., Luther-Davies, B., Moss, D. J., Monat, C. and Grillet, C., "Mid-infrared nonlinear optical response of Si-Ge waveguides with ultra-short optical pulses," Opt. Express 23, 32202 (2015).

[17] Hon, N. K., Soref, R. and Jalali, B., "The third-order nonlinear optical coefficients of $\mathrm{Si}, \mathrm{Ge}$, and $\mathrm{Si}_{1-\mathrm{x}} \mathrm{Ge}_{\mathrm{x}}$ in the midwave and longwave infrared.," J. Appl. Phys. 110, 011301 (2011).

[18] Sinobad, M., Monat, C., Luther-Davies, B., Ma, P., Madden, S., Moss, D. J., Mitchell, A., ALlioux, D., Orobtchouk, R., Boutami, S., Hartmann, J. M., Fedeli, J. M. and Grillet, C., "Mid-infrared octave spanning supercontinuum 
generation to $8.5 \mu \mathrm{m}$ in silicon-germanium waveguides," Optica 5, 360 (2018).

[19]Lu, F. and Knox, W. H., "Generation, characterization, and application of broadband coherent femtosecond visible pulses in dispersion micromanaged holey fibers," J. Opt. Soc. Am. B 23, 1221 (2006).

[20] Wong, G. K. L., Zang, L., Kang, M. S. and Russell, P. S. J., "Measurement of group-velocity dispersion of Bloch modes in photonic-crystal-fiber rocking filters," Opt. Lett. 35, 3982-4 (2010).

[21]Pan, J., Huo, Y., Yamanaka, K., Sandhu, S., Scaccabarozzi, L., Timp, R., Povinelli, M. L., Fan, S., Fejer, M. M. and Harris, J. S., "Aligning microcavity resonances in silicon photonic-crystal slabs using laser-pumped thermal tuning," Appl. Phys. Lett. 92, 90-93 (2008).

[22]Raineri, F., Cojocaru, C., Raj, R., Monnier, P. and Levenson, A., "Tuning a two-dimensional photonic crystal resonance via optical carrierinjection," Opt. Lett. 30, 64-66 (2005).

[23] Grillet, C., Monat, C., Smith, C. L., Lee, M. W., Tomljenovic-Hanic, S., Karnutsch, K. and Eggleton, B. J., "Reconfigurable photonic crystal circuits," Laser Photonics Rev. 4, 192-204 (2010).

[24]Bedoya, A. C., Domachuk, P., Grillet, C., Monat, C., Magi, E. C., Li, E. and Eggleton, B. J., "Reconfigurable photonic crystal waveguides created by selective liquid infiltration," Opt. Express 20, 11046-11056 (2012).

[25] Sinobad, M., Della Torre, A., Luther-Davies, B., Ma, P., Madden, S., Debbarma, S., Vu, K., Moss, D. J., Mitchell, A., Hartmann, J. M., Fedeli, J. M., Monat, C. and Grillet, C., "Dispersion trimming for mid-infrared supercontinuum generation in a hybrid chalcogenide/silicon-germanium waveguide," J. Opt. Soc. Am. B 36, 98-104 (2019).

[26] Bulla, D. A. P., Wang, R. P., Prasad, A., Rode, A. V., Madden, S. and Luther-Davies, B., "On the properties and stability of thermally evaporated Ge-As-Se thin films," Appl. Phys. A Mater. Sci. Process. 96, 615-625 (2009).

[27] Dantanarayana, H. G., Abdel-Moneim, N., Tang, Z., Sojka, L., Sujecki, S., Furniss, D., Seddon, A. B., Kubat, I., Bang, O. and Benson, T. M., "Refractive index dispersion of chalcogenide glasses for ultra-high numerical-aperture fiber for mid-infrared supercontinuum generation," Opt. Mater. Express 4, 1444 (2014). 